\title{
TICs in the Geometry Education: Proposals for Change the Content Approach
}

\section{SIGRADI2018 TECHNOPOLITICAS \\ xxii congresso da sociedade iberoamericana de gráfica digital 22th conference of the iberoamerican society of digital graphics 07|08|09|novembro|2018 iau usp | são carlos | sp br}

\author{
Bianca M. F. Leal \\ Programa de Pós-Graduação em Arquitetura - Universidade Federal do Rio de Janeiro, \\ PROARQ - FAU/UFRJ | Brazil | bleal.arq@gmail.com \\ Mônica Santos Salgado \\ Programa de Pós-Graduação em Arquitetura - Universidade Federal do Rio de Janeiro, \\ PROARQ - FAU/UFRJ | Brazil | monicassalgado@ufrj.br
}

\begin{abstract}
The use of technology instigates student interest. Thus, the possibilities offered by the technologies should be presented to students during their professional training. This paper aims to propose to teachers a change in the approach of 'geometry' contents through the use of BIM, Augmented Reality, Virtual Reality, Rapid Prototyping and Digital Fabrication. The methodology chosen was Systematic Review of Literature and literature review without metaanalysis. It presents an overview of the applicability of such technologies in teaching, as well as possibilities little explored. The results indicate that 'geometry' teaching can benefit from the great potential offered by Information and Communication Technologies.
\end{abstract}

Keywords: Teaching of 'geometry'; BIM; Augmented Reality; Virtual reality; Rapid Prototyping.

\section{INTRODUÇÃO}

O uso de tecnologias digitais traz efeitos variados na vida cotidiana da sociedade, da arquitetura e do meio acadêmico. Contudo, observa-se que as práticas em sala de aula nem sempre acompanham o ritmo das inovações, gerando um descompasso entre a vivência acadêmica e a experiência fora da universidade - com intenso uso de tecnologias digitais (Carvalho \& Savignon, 2012; TPE \& Instituto Inspirare, 2014).

As tecnologias digitais exercem fascínio nos indivíduos e, por esse motivo, pode agir como um catalisador do interesse pelos estudos (Braga et al., 2012). Assim, tornase necessário repensar 0 processo de ensinoaprendizagem para que as possibilidades oferecidas pelas novas tecnologias digitais sejam apresentadas aos estudantes de arquitetura durante sua formação profissional. Acredita-se que a estratégia a ser adotada deve ir além da inclusão de disciplinas que visam o ensino de softwares.

As Tecnologias de Informação e Comunicação (TICs) utilizadas como recorte da pesquisa foram Building Information Modeling (BIM), Realidade Aumentada (RA), Realidade Virtual (RV), Prototipagem Rápida (PR) e Fabricação Digital (FD). O BIM é apontado como uma das alternativas que está transformando a construção civil e, segundo Eastman et al. (2014), se refere uma atividade humana que gerencia e produz dados da construção ao longo de todo o seu ciclo de vida. De acordo com Braida, Colchete Filho \& Maya-Monteiro (2006), RA e RV também trazem possibilidades para o ensino de arquitetura, análise de projeto, análise estrutural, entre outros. Por fim, Ferracane (2015) cita que um dos avanços tecnológicos fundamentais é a impressão 3D - também conhecida como PR e FD -, que se refere à transferência de dados de um modelo digital para uma máquina de controle numérico (CNC) para produção de elementos em escala reduzida (PR) ou em escala real (FD) (Pupo, 2016).

Ao falar sobre tecnologia, Behzadan, Vassigh \& Mostafavi (2016, p. 266, tradução nossa) expressam que "os instrutores devem treinar e educar os alunos com as últimas e melhores ferramentas e métodos para preparálos para futuras carreiras avançadas que estão se tornando cada vez mais dependentes de tecnologia". Desse modo, entende-se que para efetivamente despertar o interesse dos futuros profissionais pelas possibilidades oferecidas pelas tecnologias não seria suficiente apenas "ensinar" sobre tecnologia. O mais eficiente será integrar tais métodos em novas práticas didáticas a serem adotadas no ensino dos diferentes conteúdos em arquitetura.

Nesse sentido, esse artigo apresenta uma parte dos resultados obtidos com a pesquisa de mestrado que teve por objetivo identificar de que forma as tecnologias digitais (BIM, RA, RV, PR e FD) podem ser incorporadas às práticas didáticas adotadas pelos docentes do ensino de arquitetura e, considerando as áreas de conhecimento definidas pelas Diretrizes Curriculares Nacionais (DCN) para o curso de graduação em Arquitetura e Urbanismo. Logo, o artigo possui o objetivo de propor aos professores uma mudança na abordagem dos conteúdos de 'geometria' através do uso de BIM, RA, RV, PR e FD. 
As DCN de 2010 (MEC, 2010, art. 6ㅇ) apresentam grandes áreas do saber para os cursos de Arquitetura e Urbanismo no país. Esse trabalho aborda a área 'desenho e meios de representação e expressão', que passa a ser mencionada nessa pesquisa como área de 'geometria'. As palavras-chave, extraídas das DCN, que representam os conteúdos a serem ensinados em 'geometria' são: habilidades de desenho, domínio da geometria, perspectiva, modelagem, maquetes, modelos e imagens virtuais (MEC, 2010, art. 5ㅇ․

\section{METODOLOGIA}

A pesquisa foi realizada em três etapas: (1) fundamentação teórica; (2) Revisão Sistemática de Literatura (RSL); (3) revisão de literatura sem metaanálise. A RSL analisou publicações até dezembro/2017, que relatam sobre ensino de 'geometria' e TICs. Os resultados da RSL permitiram vislumbrar alternativas de uso de TICs que são pouco exploradas no ensino de 'geometria'. Assim, apresenta-se um panorama sobre a aplicabilidade de tais tecnologias no ensino, bem como possibilidades pouco exploradas.

A segunda etapa da pesquisa corresponde a RSL, que utilizou como recorte amostral artigos de periódicos, congressos, teses e dissertações publicados nos últimos dez anos (2007 a 2017), que relatam sobre experiências didáticas que incorporação TICs (BIM, RA, RV, PR e FD) no ensino dos conteúdos de arquitetura e urbanismo.

As fontes de busca consultadas foram a revista Ambiente Construído, revista PARC, revista Gestão \& Tecnologia de Projetos, Biblioteca Digital Brasileira de Teses e Dissertações do IBICT, anais de congressos como TIC, GRAPHICA, ENTAC e SBQP, bem como base de dados Cum InCAD.

As palavras-chave utilizadas na busca foram termos genéricos das TICs pesquisadas nos idiomas português e inglês, são elas: BIM, Building Information Modeling, realidade aumentada, augmented reality, realidade virtual, virtual reality, prototipagem rápida, rapid prototyping, fabricação digital, digital manufacturing.

Os critérios de inclusão de dados foram: (a) trabalhos publicados no período de 2007 a 2017; (b) necessário tratar de uma experiência didática de uma ou mais TICs no ensino dos conteúdos de arquitetura e urbanismo; (c) a publicação deve abordar sobre ensino e pelo menos uma das TICs (BIM, RA, RV, PR ou FD); (d) as publicações devem estar disponíveis para consulta em meio eletrônico; (e) publicações que o professor usa TIC em sala de aula; (f) publicações que o aluno usa TIC em sala de aula desde que o propósito do uso da ferramenta seja para ensinar conteúdos de arquitetura e não ensinar a manipular um software.

Levando em consideração o critério (d) de inclusão, alguns trabalhos não foram considerados na pesquisa: anais do GRAPHICA 2009, seis artigos do TIC 2011, dois artigos do TIC 2013. Da mesma forma, os anais do TIC 2017 também não estavam disponíveis até janeiro de 2018, quando foi finalizada essa etapa da pesquisa.

Com relação ao critério de exclusão, foram desconsiderados os textos que usaram TIC para experimentação da tecnologia e não como um modo de ensino de um conteúdo de arquitetura.

A terceira etapa da pesquisa utilizou o método de revisão de literatura sem meta-análise, que aborda sobre diferentes aplicações de TICs no campo da arquitetura e urbanismo. Os artigos utilizados nessa etapa foram encontrados em diferentes bases de dados durante buscas que ocorreram ao longo da pesquisa de mestrado, e outras publicações foram detectadas durante a pesquisa de RSL da etapa dois. Os textos demonstram diferentes usos das TICs que podem ser adaptados e aplicados no meio acadêmico. Entretanto, é importante destacar que essa pesquisa não esgota todas as possibilidades de integração das tecnologias nas áreas de conhecimento de arquitetura e urbanismo, visto que as autoras não tiveram acesso a todas as experiências realizadas e, também, pelo fato da tecnologia avançar rapidamente e, dessa forma, surgirem novas oportunidades de aplicação de TICs no ensino.

Para cada experiência de uso de TIC foi atribuída um modo de utilizar a tecnologia, que foi chamado nessa pesquisa de 'recurso tecnológico'. Certos recursos tecnológicos apresentam diferentes maneiras de serem explorados, que foram denominados como 'métodos de rastreamento' do objeto virtual (alternativas). Então, por exemplo, a TIC RA pode ser utilizada de diferentes formas no ensino por meio dos seguintes recursos tecnológicos: relacionar o real com o virtual, manipulação do modelo, entre outros. Um dos 'métodos de rastreamento' do recurso 'relacionar o real com o virtual' trata-se de utilizar esse recurso por meio de marcador, GPS ou sem marcador.

\section{RSL: PANORAMA DE APLICAÇÕES DE TICS NO ENSINO DE GEOMETRIA}

A adoção das tecnologias digitais no ensino de arquitetura e urbanismo não é um tema novo. Algumas universidades desenvolveram experiências didáticas explorando algumas potencialidades que as TICs podem oferecer para o ensino de geometria.

Um desses casos foi relatado por Delatorre, Pereira \& Pupo (2013 apud Delatorre, 2014) que realizaram uma pesquisa no curso de Arquitetura e Urbanismo da UNOCHAPECÓ, Santa Catarina, que introduziu o BIM na disciplina de desenho arquitetônico em turmas do $1^{\circ}$ período do curso. Segundo os autores (p. 83), a ferramenta utilizada foi o Archicad e a "experiência limitou-se à modelagem e aos recursos de visualização" proporcionados pelo programa. Após a exposição de conteúdos teóricos, os alunos começaram a aprender os diferentes tipos de representação arquitetônica por meio da modelagem no software e extração de desenhos (plantas, cortes, vistas e fachadas). A pesquisa apontou que os estudantes sentiram mais facilidade de interpretar e desenhar a partir do modelo BIM se comparado com os demais alunos que aprenderam da maneira tradicional. Com a terceira dimensão, os alunos passam a ter maior compreensão da volumetria e a percepção espacial foi facilitada.

Checcucci, Amorim \& Pereira (2013) contam a experiência de introdução de BIM no curso noturno da 
Faculdade de Arquitetura da UFBA, Brasil. Entre os assuntos abordados pelos autores, se destaca a experiência da disciplina 'Desenho Projetivo' que trata sobre conteúdos de geometria descritiva, perspectiva linear, desenho projetivo, introdução ao desenho técnico e desenho arquitetônico. O diferencial é que o conteúdo foi ensinado por meio da ferramenta SketchUp e não pelos recursos tradicionais de desenho. Explicações, exercícios e trabalhos foram praticados na disciplina por uma abordagem $2 \mathrm{D}$ e 3D através deste programa.

Similarmente, Pontes (2013) introduziu o uso de ferramentas computacionais na disciplina 'Oficina de Fundamentação e Instrumentação' do curso noturno da UFMG, Brasil, em 2013. Primeiramente, os alunos aprendiam geometria descritiva pelo programa SketchUp por meio de aulas teóricas e "como ferramenta para representação dos sistemas de projeção cilíndrica e cônica" (Pontes, 2013, p. 570). O autor (p. 570) explica que "são trabalhados problemas típicos como a caracterização e interseção de planos no espaço e esses problemas são resolvidos tanto através do ferramental bidimensional do sistema projetivo quanto através dos facilitadores de operações do próprio software". E acrescenta que os alunos representam bidimensionalmente a partir do modelo geométrico e viceversa. No final, o processo foi repetido com desenhos a mão livre em escala. Depois, o aluno repete o exercício que havia sido trabalhado apenas geometricamente só que agora usando elementos construtivos como a "representação de um telhado e a solução da interseção das diversas águas" através do software Revit (Pontes, 2013, p. 570). Como o curso estava em andamento, o autor possuía resultados preliminares que apontaram que este experimento fez o inverso do processo tradicional, pois parte-se do espaço 3D representado e o transforma em uma representação 2D.

Florio (2007) descreveu uma experiência didática na disciplina Computação na Arquitetura no $3 \underline{0}$ ano da Universidade Presbiteriana Mackenzie, Brasil. Nessa disciplina, os alunos modelaram três edifícios no Revit (versão 8.1) com o propósito de "verificar o aprendizado das relações espaciais entre os elementos construtivos". Foram apontados como resultado a compreensão da representação 2D dos projetos, entendimento da sequência de execução da obra, redução de "erros de interpretação e articulação entre elementos construtivos no espaço" e, consequentemente, melhoria na qualidade dos projetos (Florio, 2007, n. p.).

Vaz, Andrade \& Silva (2011) introduziram TIC no ensino de Projeções Cotadas, mais especificamente ensino de telhados, no curso de Engenharia Civil da Universidade Federal do Paraná, Brasil. Nessa experiência, os autores usaram $75 \%$ das aulas para ministrar o conteúdo teórico e exercícios sobre projeções cotadas com instrumentos de desenho (esquadros, régua e compasso) e nos $25 \%$ restante, utilizaram o SketchUp onde modelaram os projetos 2D em 3D. Os autores concluem que essa abordagem facilitou a compreensão dos assuntos teóricos, melhorou a visualização espacial, motivou e aumentou o interesse do aluno pela disciplina e resultou em maior rendimento nos conteúdos trabalhados. Os autores usaram uma TIC para auxiliar no ensino de habilidades de desenho, entretanto, esse procedimento pode ser aplicado aos outros conteúdos de geometria como domínio da geometria, perspectiva, modelagem, maquetes e modelos.

Outra forma de ensinar geometria com tecnologia é usando Realidade Aumentada. Amim (2007) e Lima, Haguenauer \& Cunha (2007) citam uma experiência desenvolvida na Technische Universitat Wien, Áustria, chamada Construct 3D. Com essa ferramenta, os professores utilizam RA para ensinar matemática e geometria. Esta é uma "ferramenta de construção 3D, sem animação, num ambiente imersivo com propósitos educacionais" (Kaufmann et al., 2005 apud Lima, Haguenauer \& Cunha, 2007, n. p.). Com a ajuda de um Head Mounted Display (display montado na cabeça) e uma caneta interativa, os alunos visualizam objetos 3D e trabalham diretamente no espaço tridimensional, onde podem criar e realocar pontos ou sólidos tridimensionais e analisar a estrutura dos novos elementos criados. Segundo Amim (2007, p. 103), "a maior vantagem em utilizar RA, é que os estudantes passam a ver os objetos tridimensionais, que antes eles tinham que calcular e desenhar em um papel" e acrescenta que este método possibilita rápida compreensão das questões espaciais ensinadas se comparado com o método tradicional.

Lima, Haguenauer \& Cunha (2007) apresentam uma proposta de Cartões Marcadores Reconfiguráveis desenvolvido por Meiguins, Almeida \& Oikawa (2006) na UFPA, Brasil, utilizando o sistema ARToolKit. Os marcadores são formados por uma base e pequenos peças (cubos) que quando agrupados em uma certa configuração exibem diferentes objetos de RA. A primeira aplicação desses marcadores reconfiguráveis ocorreu em uma disciplina de Geometria Espacial. Segundo Meiguins, Almeida \& Oikawa (2006), uma das formas de utilizar os marcadores na disciplina para explicar os elementos que formam uma pirâmide, por exemplo, é montar um marcador que mostre primeiramente os vértices da pirâmide, depois, são acrescentas peças neste marcador para revelar arestas ligando os vértices e, por fim, adiciona-se mais peças para formar as faces. Sendo que a retirada de algumas peças representa a remoção de algum elemento virtual. Outra forma descrita pelos mesmos autores de utilizar os marcadores trata da proposição de exercícios, de modo que o enunciado da questão (texto, figura e dados) podem ser exibidos em RA por um marcador, enquanto os alunos utilizam outro marcador para interagir com modelos geométricos para resolver a questão proposta. Outra opção de exercício é utilizar um marcador com informações ensinadas em sala que auxiliem na resolução de uma questão.

Outra alternativa de uso de RA em geometria foi apresentada por Lima, Haguenauer \& Cunha (2007), que expõem um protótipo desenvolvido pela Escola de Belas Artes da UFRJ em conjunto com o Grupo Realidade Virtual (GRVA) do Laboratório LAMCE da COPPE-UFRJ. O protótipo é um aplicativo para estudo e visualização de seções cônicas (círculo, elipse, parábola ou hipérbole) da Geometria Descritiva por meio da Realidade Aumentada. Segundo os autores (n.p.), o aplicativo foi elaborado "através do DART - Designer's Augmented Reality Toolkit, que funciona no programa Macromedia Director MX", visualizador Monitor Based AR, um computador e webcam Creative. $\mathrm{O}$ aplicativo possui quatro módulos de visualização: (1) Épura: visualização simultânea das vistas ortogonais do objeto estudado no espaço 3D; (2)

3 
Sólido Épura: observação das vistas anteriores acrescido do "sólido que deu origem à épura"; (3) Sólido: visualização em 3D da seção cônica sem as projeções ortogonais; (4) Saber Mais: mostra a "definição geométrica da seção cônica escolhida, segundo o Teorema de Apolonius" (Lima, Haguenauer \& Cunha, 2007 , n.p.). Por último, os mesmos autores destacam que esse tipo de experimentação proporciona o desenvolvimento da Inteligência Espacial, enquanto o ensino tradicional prioriza 0 ensino através das Inteligências Linguística e Lógico-Matemática, o que resulta na dificuldade dos alunos compreenderem os temas abordados em sala.

Andrade, Goulart \& De La Cruz (2017) mostram o resultado de um Trabalho de Conclusão de Curso de Design do Centro Universitário de Volta Redonda (UniFOA), Brasil, que resultou em um livro chamado "Cadernos de Exercícios - desenho técnico com auxílio da realidade aumentada". Como o próprio nome induz, este livro contém exercícios de desenho técnico com 30 peças diferentes mostrando vistas ortográficas, um $Q R$ Code e um espaço tracejado para o aluno desenhar as peças em perspectiva. Com 0 aplicativo Augment instalado no smartphone ou tablet, o aluno captura o $Q R$ Code e visualiza a resposta do exercício através da peça em 3D que aparece na tela do celular/tablet e pode ser manipulada (rotacionar e mexer no zoom). Segundo os autores (n.p), o aplicativo Augment foi "escolhido por oferecer licença educacional gratuita". Vale destacar que qualquer usuário que faça um login pode inserir modelos nesse aplicativo. Dessa forma, o Augment demostra ser um recurso de fácil acesso aos professores que queiram utilizar RA em sala de aula e para os alunos que conseguem encontrar com facilidade o aplicativo no App Store ou Play Store.

Markusiewicz \& Słyk (2015) implementaram Realidade Aumentada na disciplina Computer-Aided Modeling no curso de arquitetura da Warsaw University of Technology, Polônia, nos anos acadêmicos de 2013/2014 e 2014/2015. O principal foco dos autores foi o ensino de RA para os alunos usarem em apresentações de projeto. Do ponto de vista dos autores, essa abordagem contribuiu para os alunos utilizem diferentes meios de expressarem seu trabalho - como incluir vídeo, som, texto, imagem e modelo digital em apresentações -, além de permitir que os expectadores interagem e explorem de maneira mais efetiva o modelo arquitetônico projetado. Os programas ensinados foram Metaio Creator, Metaio Cloud e aplicativo Junaio nos anos 2013/2014 e Unity 3d com Vuforia plugin, SDK e Rhinoceros nos anos $2014 / 2015$.

Exercício acadêmico utilizando Fabricação Digital é uma das possibilidades exploradas no ensino de geometria. Quintella, Ferreira \& Florêncio (2016) apresentam exemplos de pavilhões temporários desenvolvidos por meio de fabricação digital por alunos e professores em uma experiência acadêmica realizada em 2015 na FAUUFRJ. O exemplo que se destaca no ensino de geometria é o The Butterfly Gallery criado por iniciativa do PROARQ/FAU-UFRJ usando os recursos disponíveis no LAMO-3D - Laboratório da FAU-UFRJ. O pavilhão instigou a reflexão teórica e experimental sobre o "papel da geometria descritiva no contexto contemporâneo" (Quintella, Ferreira \& Florêncio, 2016, p. 323). As ferramentas de modelagem 3D utilizadas foram Rhinoceros e o plugin Grasshopper. O processo didático consistiu em workshop sobre desenho paramétrico, linguagem de programação e uso de algoritmos e, depois, são apresentadas técnicas de fabricação digital, principalmente corte a laser.

Borges et al. (2015) utilizaram artefatos metálicos de edificação de interesse patrimonial para realizar análises de geometria plana (entes geométricos, concordância, simetria e proporção), posteriormente, modelar, fazer o planejamento (definição do material e modo de montagem das partes) e corte das peças. O programa vetorial de modelagem usado foi o AutoCAD e o software para corte a laser foi o Laser CA. Esse experimento ocorreu na UFPel, Brasil, e os autores destacam que este exercício aperfeiçoou o planejamento de métodos de representação digital, promoveu maior apropriação técnica para a reprodução de modelos físicos e reprodução de formas complexas, além de melhorar a compreensão das relações de proporção de elementos.

Com base no universo pesquisado, o Quadro 1 apresenta a consolidação das experiências aqui listadas, destacando os recursos tecnológicos, os métodos de rastreamento do objeto virtual (alternativas) adotadas para cada solução proposta, os conteúdos de geometria definidos nas DCN de 2010 - que foram ensinados e o autor que citou a experiência. Percebe-se que houve uma predominância do recurso tecnológico 'interação com o modelo e o projeto' em relação ao BIM, 'manipulação do modelo virtual' quanto à RA e 'materialização das soluções/estudo da forma' sobre PR/FD. A predominância desses recursos tecnológicos talvez se deva pela facilidade de utilização deles em relação ao conteúdo que foi abordado nas experiências citadas.

\section{REVISÃO DE LITERATURA SEM META- ANÁLISE: POSSIBILIDADES POUCO EXPLORADAS}

Existem possibilidades pouco exploradas de uso de BIM no ensino de disciplinas ligadas à geometria. Borges (2016) trata sobre o desenvolvimento de competências de representação gráfica em uma experiência com uso de um programa paramétrico de modo a identificar aspectos relacionados ao ensino de expressão gráfica e sua interação com o processo de projeto em um curso de engenharia mecânica. Seguindo esse raciocínio, o professor pode usar a visualização tridimensional do modelo BIM e a documentação de projeto para ensinar desenho de arquitetura, mostrando o que cada representação simboliza no modelo, bem como princípios de perspectiva. A demonstração pode ser feita pela própria interface de software BIM de arquitetura como por aplicativos como BIMx ou A360. Sobre isso, Checcucci (2014, p. 182) alerta que além da representação, o "modelo será utilizado para diversas outras atividades [...]. Neste sentido, é importante que o estudante tenha consciência sobre os usos futuros do modelo de forma a poder participar do contexto amplo do BIM no ciclo de vida da edificação". 
Quadro 1: Consolidação das experiências didáticas de uso de TICs no ensino de geometria. Fonte: Autores.

\begin{tabular}{|c|c|c|c|c|}
\hline TIC & $\begin{array}{c}\text { Recurso } \\
\text { Tecnológico }\end{array}$ & $\begin{array}{c}\text { Método de } \\
\text { rastreamento }\end{array}$ & Conteúdo de geometria & Autor (ano) \\
\hline \multirow{4}{*}{ BIM } & \multirow{4}{*}{\multicolumn{2}{|c|}{$\begin{array}{l}\text { Interação com o modelo e o } \\
\text { projeto }\end{array}$}} & Habilidades de desenho; Modelagem & $\begin{array}{l}\begin{array}{l}\text { Delatorre, Pereira \& Pupo (2013 apud } \\
\text { Delatorre, 2014) }\end{array} \\
\end{array}$ \\
\hline & & & $\begin{array}{l}\text { Habilidades de desenho; Domínio da } \\
\text { geometria; Perspectiva; Modelagem; } \\
\text { Imagens virtuais. }\end{array}$ & Checcucci, Amorim \& Pereira (2013) \\
\hline & & & $\begin{array}{l}\text { Habilidades de desenho; Domínio da } \\
\text { geometria; Modelagem; Imagens virtuais. }\end{array}$ & Pontes (2013) \\
\hline & & & $\begin{array}{l}\text { Habilidades de desenho; Modelagem; } \\
\text { Modelos; Imagens virtuais. }\end{array}$ & Florio (2007) \\
\hline \multirow{5}{*}{ RA } & \multirow{3}{*}{$\begin{array}{l}\text { Manipulação } \\
\text { do modelo } \\
\text { virtual }\end{array}$} & \multirow{3}{*}{ Sem marcador } & Habilidades de desenho & Vaz, Andrade \& Silva (2011) \\
\hline & & & Habilidades de desenho;Perspectiva & Andrade, Goulart \& De La Cruz (2017) \\
\hline & & & Modelagem; Imagens virtuais. & Markusiewicz \& Słyk (2015) \\
\hline & \multirow{2}{*}{$\begin{array}{l}\text { Visualização } \\
\text { do modelo }\end{array}$} & \multirow{2}{*}{$\begin{array}{l}\text { Por meio de } \\
\text { marcador }\end{array}$} & \multirow[t]{2}{*}{ Domínio da geometria } & $\begin{array}{l}\text { Meiguins, Almeida \& Oikawa (2006 apud } \\
\text { Lima, Haguenauer \& Cunha, 2007) }\end{array}$ \\
\hline & & & & Lima, Haguenauer \& Cunha (2007) \\
\hline FD & \multirow{2}{*}{\multicolumn{2}{|c|}{$\begin{array}{l}\text { Materialização das } \\
\text { soluções/estudo da forma }\end{array}$}} & $\begin{array}{l}\text { Domínio da geometria; Modelagem; } \\
\text { Modelos }\end{array}$ & Quintella, Ferreira \& Florêncio (2016) \\
\hline PR & & & $\begin{array}{l}\text { Domínio da geometria; Maquetes; } \\
\text { Modelos. }\end{array}$ & Borges et al. (2015) \\
\hline
\end{tabular}

Em sua pesquisa, Checcucci (2014, p. 190) sugere

o ensino-aprendizagem das normas, da criação das diferentes pranchas da edificação e do desenho técnico pode ser realizado diretamente utilizando um programa BIM, pois além de permitirem a representação precisa da edificação também agregam outros recursos de desenho, similares aos que os programas $C A D$ tradicionais (editores de desenho 2D e modeladores geométricos - 3D) apresentam.

Além da alternativa de ensinar habilidades de desenho, conforme citado acima, o professor também pode utilizar programas BIM para ensinar modelagem e desenvolvimento de imagens virtuais. Nesses casos, os alunos explorariam as possibilidades das ferramentas BIM no que diz respeito a modelagem de modelos BIM e criação de perspectivas aéreas, cavaleira, isométrica, cônica, etc. sob a orientação do professor.

Outra alternativa para o ensino de geometria utilizando TICs é o uso de realidade aumentada. Essa TIC pode ser explorada no material didático como foi feito por Behzadan, Vassigh \& Mostafavi (2016). Os autores testaram um experimento nomeado $A R$ Magic Book na University of Central Florida, EUA. Nesse estudo, as imagens, tabelas e diagramas do livro funcionavam como um marcador, portanto, quando dispositivos portáteis habilitados a web capturavam esses marcadores, informações digitais (vídeos, sons, imagens (2D) e modelos geométricos (3D)) se sobrepunham no livro. Com isso, a RA pode ser usada para potencializar a aprendizagem com o material didático sobre qualquer conteúdo.

Amim (2007) relata um estudo feito por Kuo et al. (2004) sobre a aplicação de Realidade Aumentada no ensino de arquitetura sustentável na National Cheng Kung University, Taiwan. Nesse caso, a RA foi utilizada como um meio para exploração do jardim ecológico da universidade. Através de um tablet, os alunos visualizavam o sistema de reaproveitamento de água do jardim e informações 2D, 3D e textos ficavam sobrepostas ao cenário real, ajudando a compreender o mecanismo do campus sustentável. Além disso, era possível interagir em tempo real fazendo anotações e observações na camada virtual e os usuários podiam circular com o tablet em um eixo determinado para adquirir informações dos edifícios circundantes. Apesar do professor ter utilizado essa TIC para ensinar conforto ambiental, essa abordagem também pode ser explorada no ensino de geometria.

Gonçalves (2013) desenvolveu uma atividade com os programas Photoshop e Illustrator para analisar fotos a partir dos conceitos de perspectiva em uma turma de Produção Multimídia. Nessas imagens, os alunos identificavam o ponto de fuga, linha do horizonte, linha de terra e, em seguida, inseriam uma outra foto em perspectiva na imagem que estava sendo trabalhada. De forma semelhante, a RA pode ser utilizada para auxiliar no ensino de perspectiva através de um aplicativo que utiliza uma câmera para ler a geometria do ambiente e, então, identifica na imagem capturada a linha do horizonte e o(s) ponto(s) de fuga. Após uma aula teórica, o professor pode utilizar essa ferramenta para mostrar como funciona o processo de confecção de perspectiva que está presente nas cenas vistas todos os dias em qualquer lugar. $\mathrm{E}$, desse modo, o docente consegue também desenvolver atividades em que os alunos façam desenhos ao ar livre de modo que a ferramenta ajude a desenvolver as perspectivas corretamente.

Da mesma forma que as outras TICs, a realidade virtual também pode ser explorada no ensino de geometria. Jacques et al. (2001) criaram um ambiente de aprendizagem hipermídia que mescla modelos virtuais, animação 2D e textos explicativos para auxiliar os alunos a superarem problemas de compreensão de Geometria Descritiva. Na página criada, os autores (p. 420) demonstram uma animação com a planificação do sistema épura e também desenvolveram um exercício em épura com animação 2D que simulava "uma situação usual em sala de aula, onde um professor resolve um exercício no quadro negro, descrevendo os seus passos até a solução". Esse experimento pode ser realizado em um ambiente imersivo por meio da realidade virtual no qual o aluno se encontra virtualmente dentro de um modelo tridimensional do plano obliquo. Nesse ambiente, o aluno pode escolher diferentes objetos para serem 
projetados nos planos, e ao selecionar um deles, inicia uma animação mostrando o passo a passo do processo de planificação do objeto até chegar no resultado final em épura. Outro cenário imersivo seria a realização de exercícios em épura em que é dado uma questão e aparece uma janela com opções para o aluno resolver virtualmente o problema e, em seguida, tem uma opção para visualização da resposta final por meio de animação que mostra os passos para solução da questão.

Keller \& Schreiber (1999) apresentam um software educacional denominado GEO-3D que opera como uma ferramenta de ensino de poliedros, conteúdo de geometria espacial, e desenvolvido para alunos de ensino fundamental. O GEO-3D possui um ambiente composto por cinco modalidades: tutorial, exercícios e prática, simulação, jogo educacional e hibrida. Partindo da proposta de jogo educacional descrita pelos autores, o ensino de geometria e perspectiva pode ser apropriado por meio de exercícios em um ambiente de RV em formato de jogo. Assim, pode ser criado um jogo composto por fases que são vencidas conforme ocorre a interação do aluno com o ambiente proposto e conforme as questões selecionadas forem respondidas corretamente em cada fase. Ao selecionar uma opção de resposta, o aluno é encaminhado para a próxima fase se ela estiver correta. Caso a resposta esteja errada, o estudante é direcionado para um outro ambiente de aprendizagem. Nesse ambiente será mostrado o resultado correto do exercício e a justificativa da resposta, em seguida, o estudante deve responder outra questão de mesmo nível e sobre o mesmo conteúdo para verificação do aprendizado. Caso ele acerte a resposta, passará para a próxima fase, caso erre, novamente aparecerá a resposta correta e um novo exercício do mesmo conteúdo. Quanto mais próximo do fim do jogo, maior o nível de dificuldade das perguntas. Ao final do jogo aparecerá o tempo total do percurso e seu desempenho. O estudante poderá fazer outas tentativas para melhorar seu desempenho e tempo de percurso e comparar com seus colegas de turma.

Quadro 2: Consolidação das possibilidades pouco exploradas de uso de TICs no ensino de geometria. Fonte: Autores.

\begin{tabular}{|c|c|c|c|c|c|}
\hline TIC & $\begin{array}{c}\text { Recurso } \\
\text { tecnológico }\end{array}$ & \multicolumn{2}{|c|}{ Método de rastreamento } & Conteúdos de geometria & Autor (ano) \\
\hline \multirow[b]{2}{*}{ BIM } & \multirow{2}{*}{\multicolumn{3}{|c|}{ Interação com o modelo e o projeto }} & Habilidades de desenho; Perspectiva & Borges (2016) \\
\hline & & & & $\begin{array}{l}\text { Habilidades de desenho; Modelagem; } \\
\text { Imagens virtuais. }\end{array}$ & Checcucci (2014) \\
\hline \multirow{3}{*}{ RA } & \multirow{3}{*}{$\begin{array}{l}\text { Relacionar o } \\
\text { real com o } \\
\text { virtual }\end{array}$} & \multirow{2}{*}{$\begin{array}{l}\text { Marcador } \\
\text { ou GPS }\end{array}$} & \multirow{3}{*}{$\begin{array}{c}\text { Textos, } \\
\text { imagens } \\
\text { e/ou vídeos } \\
\text { informativos }\end{array}$} & Todos conteúdos & $\begin{array}{l}\text { Behzadan, Vassigh \& } \\
\text { Mostafavi (2016) }\end{array}$ \\
\hline & & & & Domínio da geometria; Perspectiva & $\begin{array}{c}\text { Kuo et al. (2004 apud Amim, } \\
\text { 2007) }\end{array}$ \\
\hline & & $\begin{array}{c}\text { Sem } \\
\text { marcador }\end{array}$ & & Habilidades de desenho; Perspectiva & Gonçalves (2013) \\
\hline RV & \multicolumn{3}{|c|}{ Interação com ambiente virtual } & $\begin{array}{l}\text { Habilidades de desenho; Domínio da } \\
\text { geometria; Perspectiva }\end{array}$ & $\begin{array}{l}\text { Jacques et al. (2001); Keller } \\
\text { \& Schreiber (1999); Seabra } \\
\text { \& Santos (2005) }\end{array}$ \\
\hline $\begin{array}{l}\text { PR/ } \\
\text { FD }\end{array}$ & \multicolumn{3}{|c|}{$\begin{array}{l}\text { Materialização das soluções/estudo da } \\
\text { forma }\end{array}$} & $\begin{array}{l}\text { Habilidades de desenho; Domínio da } \\
\text { geometria; Modelagem; Maquetes; } \\
\text { Modelos; Imagens virtuais }\end{array}$ & (1) \\
\hline
\end{tabular}

Outro uso de realidade virtual foi apresentado por Seabra \& Santos (2005). Os autores exibem o projeto de uma ferramenta para ser utilizada em sala de aula no ensino de Geometria Descritiva e Desenho. Segundo os autores (p. 118), a ferramenta executa "construções geométricas tridimensionais, a qual permitirá a criação de situações espaciais com pontos, retas, planos e suas projeções, conforme os movimentos da mão do usuário (usualmente, o professor)" e acrescenta que "a interface para criação e manipulação dos elementos geométricos será gestual”. O usuário poderá criar pontos, retas, planos, sólidos primitivos, selecionar e excluir objetos, rotacionar 0 sistema de coordenadas e salvar e abrir cenários construídos. Além do ensino de geometria descritiva e de desenho, conforme sugestão dos autores, esse protótipo também pode ser usado como uma ferramenta para o ensino de perspectivas.

A prototipagem rápida é uma tecnologia que também pode ser utilizada para ensinar geometria. Uma possibilidade ainda não pouco explorada no ensino com uso de PR refere-se ao uso de protótipos durante uma explicação teórica e em exercícios de geometria descritiva de modo que os alunos de posse dos modelos desenhem as vistas ortogonais no $1 \underline{0}$ e $3 \underline{0}$ diedros. Outra alternativa seria partir do desenho de vistas ortográficas para os alunos construírem um modelo geométrico e fazer a prototipagem do modelo. Nesse segundo caso, além de ensinar conceitos de geometria, o professor também desenvolve nos alunos a habilidade de modelagem, criação de imagens virtuais, modelos e maquetes.

De acordo com o universo pesquisado, o Quadro 2 reúne as experiências relacionadas especificamente às possibilidades para o ensino de geometria descritiva, e também destaca os recursos tecnológicos e os métodos de rastreamento do objeto virtual (alternativas) para cada solução proposta, os conteúdos de geometria que foram ensinados e o autor usado como referência. Ao observar os quadros nota-se uma variedade de recursos tecnológicos, proporcionados pelas TICs, que podem ser utilizadas pelo professor. 


\section{DISCUSSÃO}

Ao analisar os Quadros 1 e 2 percebe-se que os recursos tecnológicos citados nas experiências com BIM e PR/FD foram os mesmos: 'interação com o modelo e o projeto' e 'materialização das soluções/estudo da forma', consecutivamente. Já RA apresentou uma maior variedade de recursos quando comparado às outras TICs: 'visualização do modelo', manipulação do modelo virtual' e 'relacionar o real com o virtual'.

O Quadro 3 apresenta uma análise sobre a aplicabilidade das tecnologias digitais pesquisadas no ensino dos conteúdos obrigatórios das disciplinas referentes ao ensino de "geometria" nos cursos de arquitetura e urbanismo. Observa-se que as TICs consideradas nessa pesquisa contemplaram em boa parte os conteúdos obrigatórios do ensino de geometria definidos pelas Diretrizes Curriculares e, assim, constatar se BIM, RA, RV, PR e FD podem ser adotadas pelos docentes responsáveis.

Quadro 3: Verificação dos conteúdos em relação as TICs

\begin{tabular}{|c|c|c|c|c|c|c|c|c|}
\hline \multirow{2}{*}{$\begin{array}{l}\text { Conteúdos } \\
\text { de } \\
\text { 'geometria' }\end{array}$} & \multicolumn{4}{|c|}{ RSL } & \multicolumn{4}{|c|}{$\begin{array}{l}\text { Revisão de } \\
\text { literatura sem } \\
\text { meta-análise }\end{array}$} \\
\hline & $\sum$ & $\llbracket$ & $\vec{x}$ & 品 & $\underline{\underline{m}}$ & $\underset{\nwarrow}{\llbracket}$ & 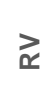 & $\frac{0}{\frac{0}{\alpha}}$ \\
\hline $\begin{array}{l}\text { Habilidades } \\
\text { de desenho }\end{array}$ & $\checkmark$ & $\checkmark$ & $x$ & $x$ & $\checkmark$ & $\checkmark$ & $\checkmark$ & $\checkmark$ \\
\hline $\begin{array}{ll}\text { Domínio } & \text { da } \\
\text { geometria } & \end{array}$ & $\checkmark$ & $\checkmark$ & $x$ & $\checkmark$ & $\mathrm{X}$ & $\checkmark$ & $\checkmark$ & $\checkmark$ \\
\hline Perspectiva & $\bar{\checkmark}$ & $\checkmark$ & $\mathrm{x}$ & $\mathrm{x}$ & $\checkmark$ & $\bar{\checkmark}$ & $\checkmark$ & $\mathrm{X}$ \\
\hline Modelagem & $\checkmark$ & $\checkmark$ & $\mathrm{X}$ & $\checkmark$ & $\checkmark$ & $\checkmark$ & $\mathrm{X}$ & $\checkmark$ \\
\hline Maquetes & $\mathrm{X}$ & $\mathrm{X}$ & $X$ & $\checkmark$ & $\mathrm{X}$ & $\checkmark$ & $\mathrm{X}$ & $\checkmark$ \\
\hline Modelos & $\checkmark$ & $\mathrm{X}$ & $\mathrm{X}$ & $\checkmark$ & $\mathrm{X}$ & $\checkmark$ & $\mathrm{X}$ & $\checkmark$ \\
\hline $\begin{array}{l}\text { Imagens } \\
\text { virtuais }\end{array}$ & $\checkmark$ & $\checkmark$ & $x$ & $x$ & $\checkmark$ & $\checkmark$ & $x$ & $\checkmark$ \\
\hline
\end{tabular}

Ao analisar as linhas do quadro nota-se que todos os conteúdos analisados utilizaram pelo menos dois recursos tecnológicos como BIM, RA, RV, PR e FD, evidenciando o suporte dado por essas tecnologias no ensino de geometria.

Ao verificar cada TIC separadamente, observa-se que, dentre os artigos pesquisados, certas tecnologias não foram associadas à alguns conteúdos de 'geometria', como são os casos marcados com ' $X$ ' vermelho, são eles: (a) ensino de maquetes com BIM; (b) ensino de modelagem, maquetes, modelos e imagens virtuais com RA; e (c) ensino de perspectiva com PR e FD. Pode-se inferir que determinados conteúdos poderão sofrer mudanças a partir da adoção das novas tecnologias no ensino de geometria, uma vez que as tecnologias digitais oferecem alternativas que podem transcender as práticas didáticas tradicionais.

\section{CONCLUSÃO}

A pesquisa revelou que BIM, RA, RV, PR e FD podem ser utilizados em sala de aula para tratar sobre desenho e meios de representação e expressão. Assim, esse artigo teve o propósito de apresentar um panorama sobre a aplicabilidade de tais tecnologias no ensino, bem como possibilidades pouco exploradas no ensino de 'geometria'.
Uma das intenções de estimular o uso de TICs na sala de aula é incentivar a compreensão de conceitos pelos alunos por meio da tecnologia, mas também instigar os interessados a estudar e aprofundar os estudos sobre as ferramentas. Desta forma, entende-se que a adoção de práticas didáticas com o uso de tecnologia refere-se à uma das estratégias para divulgar a inovação entre os futuros arquitetos. Portanto, as TICs podem ser de grande valor no processo didático na sala de aula e também como forma de encorajar os alunos a usá-las.

Por fim, os resultados indicam que o ensino de 'geometria' pode se beneficiar do grande potencial oferecido pelas TICs. Espera-se que esse trabalho possa incentivar as instituições de ensino, as coordenações e os professores a incluírem tecnologia no meio acadêmico, uma vez que a responsabilidade de incluir TICs é compartilhada por esses agentes. Acredita-se que esse estudo proporciona uma contribuição teórica para a melhoria do ensino de arquitetura e urbanismo por propor a integração das tecnologias às disciplinas do curso sem necessidade de alteração da composição curricular.

\section{AGRADECIMENTOS}

O presente trabalho foi realizado com apoio da Coordenação de Aperfeiçoamento de Pessoal de Nível Superior - Brasil (CAPES) - Código de Financiamento 001. Os autores também agradecem ao CNPq Bolsa de Produtividade em Pesquisa.

\section{REFERÊNCIAS}

Amim, R. R. (2007). Realidade aumentada aplicada à arquitetura e urbanismo. Master. Thesis, COPPE, Federal University of Rio de Janeiro, Rio de Janeiro, Brasil.

Andrade, L. S. R., Goulart, S. A. S., \& De La Cruz, P. C. H. (2017). O uso da realidade aumentada como ferramenta de ensino em expressão gráfica. Proceedings of $12^{\text {th }}$ Graphics Engineering for Arts and Design, Araçatuba. Retrieved from: https://www.even3.com.br/anais/graphica2017.

Leal, B. M. F. (2018). Propostas para o ensino dos conteúdos de arquitetura e urbanismo através de ferramentas digitais. Master. Thesis, Federal University of Rio de Janeiro, PROARQ-FAU/UFRJ, Rio de Janeiro, Brasil.

Behzadan, A. H, Vassigh, S., \& Mostafavi, A. (2016). Teaching millennials with augmented reality: cases from the U.S. education system. PARC Research in Architecture and Building Construction, v.7, n.4, 265-272. doi: http://dx.doi.org/10.20396/parc.v7i4.8649284.

Borges, L. et al. (2015). A representação de gradis metálicos como atividade didática uma conexão entre patrimônio, geometria e tecnologias de fabricação digital. Proceedings of $11^{\text {th }}$ Graphics Engineering for Arts and Design, Porto, 319328.

Borges, M. M. (2016). O uso de modeladores tridimensionais paramétricos na formação de competências de representação gráfica e raciocínio espacial no processo de projeto. Gestão \& Tecnologia de Projetos, v.11, n.1, 21-37.

Braga, R. F. et al. (2012). Estudo comparativo de toolkits de Realidade Virtual e Aumentada visando aplicação educacional. Proceedings of Workshop de Desafios da Computação Aplicada à Educação, 138-147. Retrieved from http://br-ie.org/pub/index.php/desafie/article/view/2784.

Braida, F., Colchete Filho, A., \& Maya-Monteiro, P. (2006). Inovações tecnológicas na Arquitetura e no Urbanismo: desafios para a prática projetual. Proceedings of $12^{\text {th }}$ Congresso da Associação Portuguesa para 0 Desenvolvimento das Regiões. Retrieved from: 
http://www.ufj. br/frederico braida/files/2011/02/2006 Inova \%C3\%A7\%C3\%B5es-tecnol\%C3\%B3gicas-naArquitetura.pdf.

Carvalho, R. S., \& Savignon, A. P. (2012). O professor de projeto de arquitetura na era digital: desafios e perspectivas. Gestão \& Tecnologia de Projetos, v.6, n.2, 04-13.

Checcucci, E. S. (2014). Ensino-aprendizagem de BIM nos cursos de graduação em engenharia civil e o papel da expressão gráfica neste contexto. Phd. Thesis, Federal University of Bahia, Salvador, Brasil.

Checcucci, E. S., Amorim, A. L., \& Pereira, A. P. C. (2013). Modelagem da Informação da Construção (BIM) no ensino de arquitetura. Proceedings of $17^{\text {th }}$ Sociedad Iberoamericana de Gráfica Digital, Valparaiso.

Delatorre, V. (2014). Potencialidades e limites do BIM no ensino de Arquitetura: Uma proposta de implementação. Master. Thesis, Federal University of Santa Catarina, Santa Catarina, Brasil.

Eastman, C. et al. (2014). Manual de BIM: um guia de modelagem da informação da construção para arquitetos, engenheiros, gerentes, construtores e incorporadores. Porto Alegre: Bookman.

Ferracane, M. F. (2015). Manufacturing the future: Industry 4.0. Proceedings of European Centre for International Political Economy. Retrieved from: http://ecipe.org/blog/manufacturing-the-future/.

Florio, W. (2007). Contribuições do Building Information Modeling no processo de projeto em arquitetura. Proceedings of 3rd Encontro de Tecnologia de Informação d Comunicação da Construção Civil, Porto Alegre.

Gonçalves, M. M. (2013). Ensinar perspectiva utilizando os meios digitais. Proceedings of $21^{\text {th }}$ Simpósio Nacional de Geometria Descritiva e Desenho Técnico and $5^{\text {th }}$ International Conference on Graphics for Arts and Design, Florianópolis.

Jacques, J. J. et al. (2001). Nova abordagem para o ensino de geometria descritiva básica. Proceedings of $29^{\text {th }}$ Congresso Brasileiro de Educação em Engenharia, Porto Alegre, 417422.

Keller, R., \& Schreiber, J. (1999). GEO-3D: a realidade virtual como suporte ao ensino de geometria espacial. Proceedings of $2^{\text {nd }}$ Workshop Brasileiro de Realidade Virtual, São Paulo, 182-193.

Lima, A. J. R., Haguenauer, C. J., \& Cunha, G. G. (2007). A realidade aumentada no ensino da geometria descritiva. Proceedings of $7^{\text {th }}$ Graphics Engineering for Arts and Design, GRAPHICA and $18^{\text {th }}$ Simpósio Nacional de Geometria Descritiva e Desenho Técnico, Curitiba. Retrieved from http://www.exatas.ufpr.br/portal/degraf/graphica2007a/.
Markusiewicz, J., \& Slyk, J. (2015). From shaping to information modeling in architectural education: implementation of augmented reality technology in computer-aided modeling. Proceedings of $33^{\text {th }}$ Education and Research in Computer Aided Architectural Design in Europe, Viena, 83-90.

MEC - Ministério da Educação. (2010). Resolução oㅡ 2, de 17 de junho de 2010. Institui as Diretrizes Curriculares Nacionais do curso de graduação em Arquitetura e Urbanismo, alterando dispositivos da Resolução CNE/CES nำ 6/2006. Retrieved from http://portal.mec.gov.br/index.php?option=com docman\&vie $\mathrm{w}=$ download\&alias $=5651$-rces002-10\&category_slug=junho2010-pdf\&ltemid=30192.

Meiguins, B., Almeida, I. S., \& Oikawa, M. A. (2008). Cartões Marcadores Reconfiguráveis em Ambientes de Realidade Aumentada. Proceedings of $8^{\text {th }}$ Symposium on Virtual Reality, Pará.

Pontes, M. M. (2013). Sistemas BIM no ensino de arquitetura: uma investigação do processo de ensino de geometria descritiva e desenho arquitetônico através de elementos construtivos virtuais. Proceedings of $17^{\text {th }}$ Sociedad Iberoamericana de Gráfica Digital, Valparaiso, 569-571.

Pupo, R. T. (2016). Fabricação digital. In F. Braida et al., 101 Conceitos de Arquitetura e Urbanismo na Era Digital (pp. 9899). São Paulo: ProBooks.

Quintella, I. P. C. P., Ferreira, Í. C., \& Florêncio, E. Q. (2016). Making pavilions: Os pavilhões temporários no contexto das faculdades de arquitetura e urbanismo. Proceedings of $20^{\text {th }}$ Sociedad Iberoamericana de Gráfica Digital, Buenos Aires, 318-325.

Seabra, R. D., \& Santos, E. T. (2005). Utilização de técnicas de realidade virtual no projeto de uma ferramenta $3 D$ para desenvolvimento da habilidade de visualização espacial. Revista Educação Gráfica, 9, 111-122.

TPE - Todos Pela Educação, \& Instituto Inspirare. (2014) Inovações tecnológicas na educação: contribuições para gestores públicos. Retrieved from http://www.todospelaeducacao.org.br/biblioteca/conteudotpe/1497/inovacoes-tecnologicas-na-educacao-contribuicoespara-gestores-publicos/.

Vaz, A., Andrade, A. F., \& Silva, R. (2011). Modelando coberturas no Sketchup: uma experiência didática. Proceedings of $20^{\text {th }}$ Simpósio Nacional de Geometria Descritiva e Desenho Técnico and $9^{\text {th }}$ Graphics Engineering for Arts and Design, Rio de Janeiro. 